\title{
Front Matter: Volume 9900
}

, "Front Matter: Volume 9900," Proc. SPIE 9900, Quantum Optics, 990001 (6 July 2016); doi: 10.1117/12.2244527

SPIE. Event: SPIE Photonics Europe, 2016, Brussels, Belgium 


\section{PROCEEDINGS OF SPIE}

\section{Quantum Optics}

Jürgen Stuhler

Andrew J. Shields

Editors

\section{5-7 April 2016 \\ Brussels, Belgium}

Sponsored by

SPIE

Cosponsored by

B-PHOT—Brussels Photonics Team (Belgium)

Research Foundation Flanders (Belgium)

Visit Brussels (Belgium)

Cooperating Organisations

Photonics 21 (Germany)

EOS-European Optical Society (Germany)

KTN-the Knowledge Transfer Network (United Kingdom)

Graphene Flagship (Belgium)

Photonics Public Private Partnership (Belgium)

Published by

SPIE 
The papers in this volume were part of the technical conference cited on the cover and title page. Papers were selected and subject to review by the editors and conference program committee. Some conference presentations may not be available for publication. Additional papers and presentation recordings may be available online in the SPIE Digital Library at SPIEDigitallibrary.org.

The papers reflect the work and thoughts of the authors and are published herein as submitted. The publisher is not responsible for the validity of the information or for any outcomes resulting from reliance thereon.

Please use the following format to cite material from these proceedings:

Author(s), "Title of Paper," in Quantum Optics, edited by Jürgen Stuhler, Andrew J. Shields, Proceedings of SPIE Vol. 9900 (SPIE, Bellingham, WA, 2016) Six-digit Article CID Number.

ISSN: 0277-786X

ISSN: 1996-756X (electronic)

ISBN: 9781510601451

Published by

SPIE

P.O. Box 10, Bellingham, Washington 98227-0010 USA

Telephone +1 3606763290 (Pacific Time) · Fax +1 3606471445

SPIE.org

Copyright (C) 2016, Society of Photo-Optical Instrumentation Engineers.

Copying of material in this book for internal or personal use, or for the internal or personal use of specific clients, beyond the fair use provisions granted by the U.S. Copyright Law is authorized by SPIE subject to payment of copying fees. The Transactional Reporting Service base fee for this volume is $\$ 18.00$ per article (or portion thereof), which should be paid directly to the Copyright Clearance Center (CCC), 222 Rosewood Drive, Danvers, MA 01923. Payment may also be made electronically through CCC Online at copyright.com. Other copying for republication, resale, advertising or promotion, or any form of systematic or multiple reproduction of any material in this book is prohibited except with permission in writing from the publisher. The CCC fee code is 0277-786X/16/\$18.00.

Printed in the United States of America.

Publication of record for individual papers is online in the SPIE Digital Library.

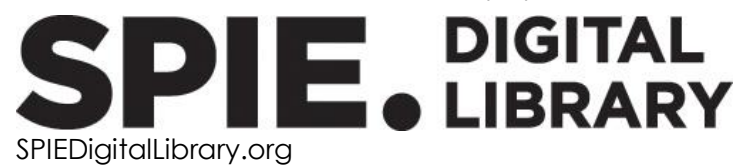

Paper Numbering: Proceedings of SPIE follow an e-First publication model, with papers published first online and then in print. Papers are published as they are submitted and meet publication criteria. A unique citation identifier (CID) number is assigned to each article at the time of the first publication. Utilization of CIDs allows articles to be fully citable as soon as they are published online, and connects the same identifier to all online, print, and electronic versions of the publication. SPIE uses a six-digit CID article numbering system in which:

- The first four digits correspond to the SPIE volume number.

- The last two digits indicate publication order within the volume using a Base 36 numbering

system employing both numerals and letters. These two-number sets start with 00, 01, 02, 03, 04, $05,06,07,08,09,0 A, 0 B \ldots$.. 0Z, followed by 10-1Z, 20-2Z, etc.

The CID Number appears on each page of the manuscript. The complete citation is used on the first page, and an abbreviated version on subsequent pages. 


\title{
Contents
}

\author{
$\checkmark$ Authors \\ vii Conference Committee
}

\section{ATOM SENSORS I}

990003 Development of a strontium optical lattice clock for the SOC mission on the ISS (Best Student Paper Award) [9900-2]

990004 Development of compact cold-atom sensors for inertial navigation [9900-3]

990007 Towards rotation sensing with a single atomic clock [9900-6]

990008 MIGA: combining laser and matter wave interferometry for mass distribution monitoring and advanced geodesy [9900-7]

\section{QUANTUM TECHNOLOGIES}

990009 The UK National Quantum Technologies Hub in sensors and metrology (Keynote Paper) [9900-8]

\section{ATOM SENSORS II}

9900 OF Optical atomic magnetometry for magnetic induction tomography of the heart [9900-14]

\section{QUANTUM COMMUNICATIONS I}

9900 0J Satellite quantum communication towards GEO distances [9900-18]

9900 OK Aerospace laser communications technology as enabler for worldwide quantum key distribution [9900-19]

$9900 \mathrm{OL}$ Encoding M classical bits in the arrival time of dense-coded photons [9900-20]

\section{QUANTUM COMMUNICATIONS II}

9900 ON Improving the coherence properties of solid-state spin ensembles via optimized dynamical decoupling [9900-23]

9900 OP Demonstration of a coexistence scheme between polarization-entangled QKD and classical data channels [9900-25] 


\section{QT APPLICATIONS AND INSTRUMENTS I}

9900 OS Initialization and measurement of nitrogen-vacancy centers in diamond with plasmonic Purcell enhancement [9900-28]

9900 OT Coherent two-photon excitation of quantum dots [9900-29]

9900 OU Free-space single-photon transistor based on Rydberg interaction [9900-30]

\section{QUANTUM INFORMATION AND INSTRUMENTS}

990012 Quantum noise in energy-efficient slow light structures for optical computing: sqeezed light from slow light [9900-38]

\section{POSTER SESSION}

990017 Modeling cavities exhibiting strong lateral confinement using open geometry Fourier modal method [9900-43]

9900 1A Maximizing the information gain of a single ion microscope using Bayes experimental design [9900-47]

9900 1B Feasibility of two-photon rotational spectroscopy on trapped HD+ [9900-48]

$99001 \mathrm{C}$ Orthogonal frequency division multiplexed quantum key distribution in the presence of Raman noise [9900-49]

9900 lE 1THz synchronous tuning of two optical synthesizers [9900-52]

$99001 G$ Compact narrow linewidth diode laser modules for precision quantum optics experiments on board of sounding rockets [9900-54] 


\title{
Authors
}

Numbers in the index correspond to the last two digits of the six-digit citation identifier (CID) article numbering system used in Proceedings of SPIE. The first four digits reflect the volume number. Base 36 numbering is employed for the last two digits and indicates the order of articles within the volume. Numbers start with 00, 01, 02, 03, 04, 05, 06, 07, 08, 09, OA, OB...0Z, followed by 10-12, 20-2Z, etc.

\author{
Aleksic, Slavisa, OP \\ Amand, L., 08 \\ Antoni-Micollier, L., 04 \\ Arnold, A. S., 09 \\ Bacco, Davide, OJ \\ Bahrani, Sima, IC \\ Bar-Gill, Nir, ON, OS \\ Barrett, B., 04 \\ Barwood, G., 03 \\ Bason, Mark G., 07, 09 \\ Battelier, B., 04 \\ Beardsley, R. P., 09 \\ Benkler, Erik, $1 \mathrm{E}$ \\ Benson, T. M., 09 \\ Bertoldi, A., 08 \\ Bianco, Giuseppe, 0J \\ Bishop, Thomas, 07 \\ Bize, S., 03 \\ Bongs, K., 03, 09 \\ Bostock, H., 09 \\ Bouyer, P., 04, 08 \\ Bowtell, R. W., 09 \\ Boyer, V., 09 \\ Brookes, M. J., 09 \\ Budker, D., ON \\ Campion, R. P., 09 \\ Canuel, B., 08 \\ Chichet, L., 04 \\ Constantin, Florin L., 1B \\ Cormier, E., 08 \\ Cross, T., 09 \\ Cruise, M. A., 09 \\ Cumming, D. R. S., 09 \\ Deans, Cameron, OF \\ Dequal, Daniele, 0J \\ Dobrovitski, V. V., ON \\ Erbert, Götz, 1G \\ Ertmer, W., 03 \\ Fang, B., 08 \\ Farfurnik, D., ON \\ Fernholz, Thomas, 07, 09 \\ Fouché, L., 04 \\ Freegarde, T., 09 \\ Freise, A., 09 \\ Fromhold, T. M., 09 \\ Fuchs, Christian, OK \\ Gaffet, S., 08 \\ Gaiarin, Simone, OJ \\ Garraway, B. M., 09
}

\author{
Geiger, R., 08 \\ Gentile, Fabio, 07 \\ Gill, P., 03, 09 \\ Gorniaczyk, Hannes, OU \\ Gregersen, Niels, 17 \\ Griffin, P. F., 09 \\ Groot-Berning, Karin, 1A \\ Hackermuller, L., 09 \\ Hamerly, Ryan, 12 \\ Harms, J., 08 \\ Hastie, J. E., 09 \\ Häyrynen, Teppo, 17 \\ $\mathrm{He}, \mathrm{W} ., \mathrm{O} 3$ \\ Hegazy, Salem F., OL \\ Hempler, N., 09 \\ Hensinger, W., 09 \\ Hentschel, Michael, OP \\ Hill, I. R., 03, 09 \\ Himsworth, M., 09 \\ Hipp, Florian, OP \\ Hofferberth, Sebastian, OU \\ Holleville, D., 03, 08 \\ Holynski, M., 09 \\ Huber, T., OT \\ Hübel, Hannes, OP \\ Hughes, J., 03, 09 \\ Hush, Michael R., 07 \\ Hussain, Sarah, OF \\ Jacob, Georg, 1A \\ Jammi, Sindhu, 07 \\ Jamshidi, Kambiz, 12 \\ Jarmola, A., ON \\ John, P., 09 \\ Kaenders, W., 03 \\ Kaushik, A., 09 \\ Kock, B. O., 09 \\ Kohfeldt, Anja, 1G \\ Krüger, P., 09 \\ Kulosa, A., 03 \\ Kürbis, Christian, $1 \mathrm{G}$ \\ Landragin, A., 04, 08 \\ Lautier, J., 04 \\ Lefèvre, G., 08 \\ Lesanovsky, Igor V., 07 \\ Le Targat, R., 03 \\ Lhermite, J., 08 \\ Li, X., 09 \\ Lien, Y.-H., 09 \\ Lisdat, Ch., 03
}


Lodewyck, J., 03

Luceri, Vincenza, 0J

Luvsandamdin, Erdenetsetseg, IG

Mabuchi, Hideo, 12

Maclean, J. O., 09

Maddox, S., 09

Malcolm, G. P. A., 09

Marmugi, Luca, OF

Marsh, J. H., 09

Mas, Hèctor, 07

Melén, Gwen, OK

Mellor, C. J., 09

Mielec, N., 08

Mirgorodskiy, Ivan, OU

Moll, Florian, OK

Morra, Ahmed E., OL

Napolitano, F., 04

Naverth, Sebastian, OK

Neuhaus, Rudolf, $1 \mathrm{E}$

Niggebaum, A., 09

Nizamani, A. H., 09

Novikov, S. V., 09

Obayya, Salah S. A., OL

Origlia, S., 03

Orucevic, F., 09

Ostermann, L., OT

Ovchinnikov, Y., 03

Paboeuf, D., 09

Pandey, Saurabh, 07

Paris-Mandoki, Asaf, OU

Parrotta, D. C., 09

Pasquazi, A., 09

Paul, D. J., 09

Peccianti, M., 09

Pelisson, S., 08

Perea-Ortiz, M., 09

Peters, Achim, $1 G$

Petrov, P., 09

Pham, L. M., ON

Plant, S., 09

Poppe, Andreas, OP

Porte, H., 04

Poschinger, Ulrich G., $1 \mathrm{~A}$

Potter, E., 09

Poulios, Konstantinos, 07

Pramod, M. S., 03

Predojević, A., OT

Prevedelli, M., 08

Prilmüller, M., OT

Puppe, Thomas, $1 \mathrm{E}$

Pyragius, Tadas, 07

Raab, Christoph, 1E

Rapaport, Ronen, OS

Rasel, E.-M., 03

Rau, Markus, OK

Razavi, Mohsen, 1C

Renzoni, Ferruccio, OF

Riis, E., 09

Riou, I., 08

Ritsch, H., OT
Rodriguez Blanco, A., 09

Rohde, Felix, 1E

Rosenberg, Itamar, OS

Ruostekoski, J., 09

Rushforth, A. W., 09

Salehi, Jawad A., 1C

Schiavon, M., OJ

Schiemangk, Max, $1 G$

Schiller, S., 03

Schmidt, Christopher, OK

Schmidt-Kaler, Ferdinand, 1A

Singer, Kilian, 1A

Singh, Y., 03, 09

Sinuco-Leon, G., 09

Smith, L., 03

Smith, P., 09

Solomon, G. S., OT

Sorel, M., 09

Stabrawa, A., 09

Sterr, U., 03

Stevenson, Robin, 07

Stuhler, Jürgen, 03, $1 \mathrm{E}$

Świerad, D., 03

Telle, Harald R., $1 \mathrm{E}$

Tomasin, M., OJ

Tränkle, Günther, $1 \mathrm{G}$

Tresp, Christoph, OU

Tropper, A., 09

Unterreitmayer, Reinhard, IE

Vallone, Giuseppe, 0J

Vasilakis, Georgios, 07

Vedovato, F., OJ

Venon, B., 03

Villoresi, Paolo, 0J

Viswam, S., 03

Vogl, Tobias, OK

Vogt, S., 03

von Klitzing, Wolf, 07

Walsworth, R. L., ON

Wang, Z. H., ON

Weihs, G., OT

Weinfurter, Harald, OK

Welch, N., 09

Wicht, Andreas, $1 G$

Wildman, R. D., 09

Williams, R. A., 09

Wolf, Sigal A., OS

Zach, Armin, $1 \mathrm{E}$

Zimmer, Christian, OU 


\section{Conference Committee}

Symposium Chairs

Francis Berghmans, Vrije Universiteit Brussel (Belgium)

Jürgen Popp, Leibniz-Institut für Photonische Technologien e.V.

(Germany)

Ronan Burgess, European Commission Photonics Unit (Belgium)

Peter Hartmann, SCHOTT, AG (Germany)

Honorary Symposium Chair

Hugo Thienpont, Vrije Universiteit Brussel (Belgium)

Conference Chairs

Jürgen Stuhler, TOPTICA Photonics AG (Germany)

Andrew J. Shields, Toshiba Research Europe Ltd. (United Kingdom)

Conference Program Committee

Christoph Becher, Universität des Saarlandes (Germany)

Oliver Benson, Humboldt-Universität zu Berlin (Germany)

Rainer Blatt, Leopold-Franzens-Universität Innsbruck (Austria)

Kai Bongs, The University of Birmingham (United Kingdom)

Philippe Bouyer, Institut d'Optique Graduate School LP2N (France) and Muquans Bordeaux (France)

Michael Jetter, Universität Stuttgart (Germany)

Christian Kurtsiefer, National University of Singapore (Singapore)

Eugene S. Polzik, Niels Bohr Institute (Denmark)

Bruno Sanguinetti, id Quantique SA (Switzerland)

Session Chairs

1 Atom Sensors I

Kai Bongs, The University of Birmingham (United Kingdom)

2 Quantum Technologies

Jürgen Stuhler, TOPTICA Photonics AG (Germany)

3 Atom Sensors II

Ulrich Eismann, TOPTICA Photonics AG (Germany)

4 Quantum Communications I

Andrew J. Shields, Toshiba Research Europe Ltd. (United Kingdom) 
5 Quantum Communications II

Martin Ward, Toshiba Research Europe Ltd. (United Kingdom)

6 QT Applications and Instruments I

Tara Liebisch, Universität Stuttgart (Germany)

7 QT Applications and Instruments II

Thomas Fernholz, The University of Nottingham (United Kingdom)

8 Quantum Information and Instruments

Ana Predojevic, University of Innsbruck (Austria) 